\title{
Correction to: Thirteen-week subcutaneous repeated dose toxicity study of butylparaben and its toxicokinetics in rats
}

\author{
Jin-Sook Bae ${ }^{1,2} \cdot$ Jung Dae Lee ${ }^{3} \cdot$ Si-Whan Song ${ }^{1} \cdot$ Ho-Chul Shin ${ }^{2} \cdot$ Yong-Kyu Choi ${ }^{4} \cdot$ Chan Young Shin ${ }^{5}$. \\ Byung-Mu Lee ${ }^{6} \cdot$ Kyu-Bong Kim ${ }^{3}$ (I)
}

Published online: 24 April 2021

๑) The Author(s), under exclusive licence to Springer-Verlag GmbH Germany, part of Springer Nature 2021

\section{Correction to: Archives of Toxicology \\ https://doi.org/10.1007/s00204-021-03037-9}

In the original publication of the article, the spelling of one of the authors' name was published with an error. The name was published as "Ho-Cheol Shin" but it should be "HoChul Shin".

The original article has been updated with the correct name.

Publisher's Note Springer Nature remains neutral with regard to jurisdictional claims in published maps and institutional affiliations.

The original article can be found online at https://doi.org/10.1007/ s00204-021-03037-9.

Kyu-Bong Kim

kyubong@dankook.ac.kr

1 Nonclinical Research Center, Chemon Inc., 240,

Nampyeong-ro, Yangji-myeon, Cheoin-gu, Yongin-si,

Gyeonggi-do 17162, Republic of Korea

2 College of Veterinary Medicine, Konkuk University, 120 Neungdong-ro, Gwangjin-gu, Seoul 05029, Republic of Korea

3 Department of Pharmacy, College of Pharmacy, Dankook University, 119 Dandae-ro, Cheonan, Chungnam 31116, Republic of Korea

4 Cosmetics Research Team, Pharmaceuticals and Medical Devices Research Department, National Institute of Food and Drug Safety Evaluation, Ministry of Food and Drug Safety, Osong 28159, Republic of Korea

5 Department of Neuroscience, School of Medicine and Center for Neuroscience Research, Konkuk University, Seoul 05029, Republic of Korea

6 College of Pharmacy, Sungkyunkwan University, Seobu-ro 2066, Changan-Ku, Gyeonggi-Do, Suwon 16418,

Republic of Korea 